\title{
Fundamentals of a New Sub-Diffraction Direct Laser Writing Method by a Combination of Stimulated Emission Depletion and Excited State Absorption
}

\author{
Christoph Wenisch*, Sebastian Engel, Stephan Gräf and Frank A. Müller \\ Otto Schott Institute of Materials Research (OSIM), Friedrich Schiller University Jena, Germany \\ ${ }^{*}$ Corresponding author's e-mail: christoph.wenisch@uni-jena.de
}

\begin{abstract}
We present a unique method of hybrid laser processing by structuring $200 \mathrm{~nm}$ thin $\mathrm{ZnO}$ films sputtered on fused silica substrates. By a combination of two pulsed ns-laser beams with different photon energies - one below and one above the $\mathrm{ZnO}$ band gap energy - the controlled independent ablation of the film devoid of any substrate damage is demonstrated. Compared to single beam ablation, we reduce the amount of debris and thermal defects at the surface at comparable laser energy conditions. To quantify the impact of this dual beam set-up on ablation quality and efficiency, several parameters like pulse delay, laser wavelength, and pulse fluence were varied. These results establish the basis for the proposed novel direct sub diffraction writing method of semiconductors by a combination of the presented principle and stimulated emission.
\end{abstract}

DOI: $10.2961 /$ jlmn.2020.03.2002

Keywords: excited state absorption, ESA based processing, stimulated emission depletion, Sub-diffraction direct laser writing, STED-lithography

\section{Introduction}

In the same way that the Abbe limit restricts the resolution of conventional optical microscopy, the diffraction limit restricts the focusability of laser radiation [1]. As a result, for direct laser structuring in an oxygen atmosphere a systemdependent proportionality factor $\mathrm{k}=0.7-1$ is implemented, which always results in resolution degradation [2]. The achievable structure sizes $d_{L S}=k \cdot \lambda / N A$ for a linear one photon absorption with the wavelength $\lambda$ and numerical aperture NA, already allows structures below 200 and $100 \mathrm{~nm}$ for visible and ultraviolet laser irradiation, respectively [2,3]. However, numerous applications in photonics, plasmonics, optoelectronics, and quantum computing require structural sizes that are not limited by the utilized wavelength and the associated limit of focusability [4-10].

Based on a top-down approach, numerous methods have already been established reaching structure sizes below $200 \mathrm{~nm}$ like electron and ion beam lithography with $\mathrm{d}_{\mathrm{LS}}<10 \mathrm{~nm}[8,11]$. However, the required vacuum and other technologically complex processing steps combined with a low throughput makes these methods very expensive, timeconsuming, and limits the size of the processed components $[12,13]$. Alternative lithographic processes are based on UV, EUV, and X-ray radiation $[14,15]$, whereby the most commonly used photolithographic technique based on optical far-field lithography uses radiation of $\lambda=193 \mathrm{~nm}$ and can produce low double digit structure sizes $[2,3]$. With optical near field lithography, structures of $d_{L S}<20 \mathrm{~nm}$ can be produced [13]. However, due to the necessity of special optics, the often required vacuum, and the limitation to surface structuring, both UV and X-ray lithography can only be used to a very limited extent $[14,15]$. Although it is possible to generate structures with a periodicity below the resolution limit (e.g., light induced periodic surface structures) by exploiting interference effects, the pattern complexity is very limited $[13,16,17]$. As a primarily non-optical structuring technique, $\mathrm{d}_{\mathrm{LS}}<20 \mathrm{~nm}$ are achieved by means of nanoimprint lithography, whereby the desired patterns must be applied to stamps first, using different (lithographic) processes in order to subsequently transfer them, as well $[13,18]$.

\subsection{Material processing based on nonlinear effects}

By focusing and deflecting the laser spot relative to the material surface, the flexibility for written structures is highly increased. In the case of non-linear threshold effects, one can take advantage of the gaussian shaped intensity profile of the laser beam. Here, only the central focal area reaches the required intensity threshold for structuring by, e.g., melt formation, sublimation, polymerization, or phase transformation $[4,7,19,20]$.

Further restriction of the spatial intensity results in nonlinear multiphoton processes in the beam center and decreases the structured area even further. This nonlinear behavior is the basis for multiphoton polymerization [21] and 3-dimensional direct laser writing inside materials with a distinct band gap like dielectrics and semiconductors [22]. In the latter case, the multi-photon absorption can be divided into two successive processes. The initial multiphoton-based excitation of, e.g., electrons, followed by a subsequent secondary excitation of these carriers like intraband absorption, avalanche effects, and coulomb-explosion. Since the effective intensity profile $\mathrm{I}_{(\mathrm{r})}{ }^{\mathrm{N}}$ is nonlinearly dependent on the number $\mathrm{N}$ of necessary photons, 3-dimensional structure sizes below the diffraction limit are possible [10,23,24].

Analogous to the resolution limit of multiphoton microscopy [25], a decreased resolution limit $\mathrm{d}_{\mathrm{MPP}}=\mathrm{k} \cdot \lambda /\left(\mathrm{NA} \cdot \mathrm{N}^{1 / 2}\right)$ can be defined for multiphoton structuring processes, which is about $200 \mathrm{~nm}$ for typical $\lambda$-values $[23,26,27]$. It becomes evident, that $d_{\text {MPP }}$ is also limited in its spatial resolution with a proportionality to $1 / \mathrm{N}^{1 / 2}$ where values of up to $\mathrm{N} \leq 10$ are 
typically used [22]. Here, two opposing processes affect the minimal structure sizes. For a material with a specific band gap, the highest possible laser wavelength should be used to increase the number of photons involved in the process. On the contrary, larger wavelengths decrease the focusability and thus, increase the focus.

\subsection{STED and ESA based processing}

Based on the stimulated emission depletion (STED) microscopy, in the last decade STED based direct write techniques were developed [28], combining photopolymerization with resolutions below the diffraction limit. Due to a carrier relaxation of the outer parts of an excitation beam, the polymerization is locally inhibited and restricted to the central region of a second, spatially and temporally aligned donut shaped beam [29]. This process, combined with threshold effects or multiphoton excitation, allows for structures in the low nm range [30]. A major drawback is the limitation to specific photo polymers or a separate transfer step to structure other classes of material [6]. The implementation of this process to directly structure (semi) conductors for commercial applications in plasmonics, photonics, quantum computing, or waveguides is not shown so far [31].

To adept the already established methods to these classes of material, we present an excited state absorption (ESA) based ablation process to better understand the excitation and relaxation mechanisms and allow to improve and extend this to zinc (II) oxide ( $\mathrm{ZnO})$.

The detailed principle of the proposed method is described in more detail elsewhere [32]. In short, the STED technology defines the optically excited region well below the diffraction limit, which is subsequently processed or modified by a separate ESA beam. Here, only the ESA based ablation is studied, separate from the subsequent relaxation process, necessary to restrict the ablation area which would allow for sub diffraction structuring. For the ESA process, an excitation beam (pump beam) promotes carriers to an excited state (e.g., conduction band, energy levels, color centers). These carriers can then be absorbed by the otherwise transmitted ESA beam and be excited even further, allowing for an increased absorbed energy for ablation. The process is spatially and temporally restricted to the superposition of, in our case, two separate laser pulses with different photon energy, one below and one above the $\mathrm{ZnO}$ band gap energy.

\section{Experimental procedure}

The samples were prepared by sputtering $200 \mathrm{~nm}$ thin $\mathrm{ZnO}$ layers (sputter time $=37 \mathrm{~min}$, pressure $=2.95 \mu \mathrm{bar}$, gas flow $=6 \mathrm{sccm}$ Ar with $2 \% \mathrm{O}_{2}$, power $=150 \mathrm{~W}$ ) on cleaned fused silica substrates with a thickness of $1 \mathrm{~mm}$ (Infrasil 301, Heraeus, Germany). The absorption was determined by UVVIS spectrometry (MCS/100-3, J\&M Analytik) using uncoated fused silica as reference. In addition, qualitative photoluminescence measurements with a HeCd-laser ( $\lambda=325 \mathrm{~nm}$, power $=1.5 \mathrm{~mW}$, IK3202R-D, Kimmon Koha $)$ as an excitation source were performed and the fluorescence was detected in a $45^{\circ}$ angle by an optical spectrometer (SP2500i, Princeton Instruments). The morphological changes after laser processing were determined by optical microscopy (VH-Z100, Keyence).

For the proposed dual beam hybrid laser process by an ESA, two Nd:YAG-lasers (SLI-10, Amplitude Systems) were used. The pump beam operates at its third harmonic with a wavelength $\lambda_{\text {pump }}=355 \mathrm{~nm}$ and a pulse width $\tau_{\text {pump }}=6.9 \mathrm{~ns}$. The ESA beam was implemented by an additional optical parametric oscillator (OPO) that emits pulses at $\lambda_{\mathrm{ESA}}=500$ and $600 \mathrm{~nm}$ with $\tau_{\mathrm{ESA}}=5.5 \mathrm{~ns}$. The laser beams were focused on the sample from opposite directions, i.e., contrary to the pump beam, the ESA beam passes through the fused silica substrate (Fig. 1).

To reduce accumulation effects like a steady sample heating, defocusing through diffraction index changes, contamination of the surface by resolidified $\mathrm{ZnO}$, or the formation of deep level defects and color centers, resulting in an altered absorption, for all measurements, single pulses were used. In order to eliminate the influence of fluctuating laser pulse energies $(\sim 8 \%)$ and inhomogeneities of the sample, the diameter of 5 independent ablation spots fabricated with identical parameters of both pulses were averaged for all performed single and dualbeam ablation measurements.

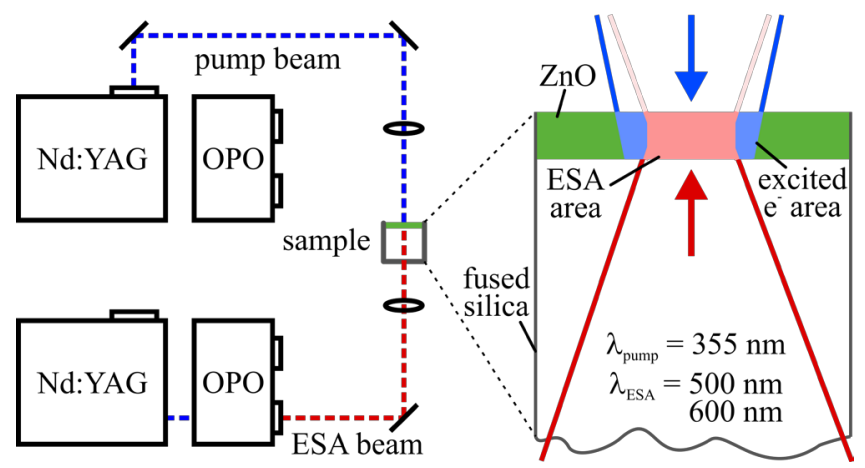

Fig. 1 Dual beam setup for ESA based ablation. The sample cross section highlights the beam directions and superimposed region for ESA based processing of the $200 \mathrm{~nm}$ thin $\mathrm{ZnO}$ layer devoid of any substrate damage.

To determine the ablation behavior of the $\mathrm{ZnO}$ layer for each laser separately, first the effect of each individual laser beam was analyzed on its own. For this purpose, the ablation diameters $\mathrm{D}$ were measured as a function of their respective pulse energy $\left(\mathrm{E}_{\text {pump }} \leq 22 \mu \mathrm{J}, \mathrm{E}_{\mathrm{ESA}}^{500} \leq 72 \mu \mathrm{J}, \mathrm{E}_{\mathrm{ESA}}^{600} \leq 88 \mu \mathrm{J}\right)$. According to the method proposed by Liu [33], the associated peak ablation threshold fluences $F_{\text {th }}$ and beam diameters $2 \mathrm{w}_{\mathrm{f}}$ were calculated (Fig. 2).

Based on the results of the single pulse interaction, the ESA based laser processing with a combining of both, the pump and ESA beam, was performed. To evaluate the impact of the ESA beam fluence $\mathrm{F}_{\mathrm{ESA}}$ and the time delay $\Delta \mathrm{t}$ between the pulses (Fig. $3 \mathrm{a}$ ) on the ESA based ablation, $\Delta \mathrm{t}$ was tuned from -10 to $500 \mathrm{~ns}$ using different values of $\mathrm{F}_{\mathrm{ESA}}$ $\left(\mathrm{F}_{\mathrm{ESA}}^{500} \leq 1.0 \mathrm{~J} / \mathrm{cm}^{2}, \mathrm{~F}_{\mathrm{ESA}}^{600} \leq 1.2 \mathrm{~J} / \mathrm{cm}^{2}\right)$ keeping the pump beam fluence $F_{\text {pump }}$ constant at the optimal determined value of $0.2 \mathrm{~J} / \mathrm{cm}^{2}$ (Fig. 4). It has to be noted that a negative temporal delay $(\Delta \mathrm{t}<0)$ implies that the ESA beam precedes the pump pulse (Fig. 1).

\section{Results and discussion}

\subsection{Optical characterization}

The optical analysis of the $\mathrm{ZnO}$ layer reveals a characteristic direct band gap at $\mathrm{E}_{\mathrm{BG}}=3.3 \mathrm{eV}$ (Fig. 3b), determined by a Tauc-plot [34], indicated by the single steep absorption edge at $\lambda \sim 400 \mathrm{~nm}$ which is in good agreement with literature values [35]. This results in a high fundamental 
absorption of the pump and a high transmittance $\mathrm{T}$ of the ESA beam [36]. With the characteristic wavelike oscillation of $\mathrm{T}$, it can be assumed that the non-transmitted light is mainly attributed to reflection losses based on interference effects, typical for layers with a thickness in the order of the applied wavelength [37]. The same applies to the photoluminescence, which shows both a well-known band edge emission in the range of $\lambda \sim 400 \mathrm{~nm}$ and a broad deep level emission $(\lambda \sim 500-800 \mathrm{~nm})[38]$.

\subsection{Ablation thresholds}

The ablation threshold energies $E_{\text {th }}$ and the focal beam diameters $2 \mathrm{w}_{\mathrm{f}}$ at the sample surface were determined for the pump and the ESA beams using the method proposed by Liu [33]. For this purpose, $\mathrm{D}^{2}$ was plotted versus $\mathrm{E}$ in a semilog plot and the linear fit was extrapolated to zero (Fig. 2). Consequently, the respective peak ablation threshold fluences $\mathrm{F}_{\text {th }}=2 \mathrm{E}_{\text {th }} /\left(\pi \cdot \mathrm{w}_{\mathrm{f}}^{2}\right)$ were determined.

$\mathrm{E}_{\text {th }}$ for the single pulse ablation of the ESA beams are about one order of magnitude larger when compared to the pump beam. This can be explained by the deviating photon energies that require multiphoton processes in the case of the ESA beam $\left(\mathrm{E}_{\mathrm{ESA}}<\mathrm{E}_{\mathrm{BG}}\right)$ and allow to directly excite electrons to the conduction band (fundamental absorption) in the case of the pump beam $\left(\mathrm{E}_{\text {pump }}>\mathrm{E}_{\mathrm{BG}}\right)$. The slopes for the ESA beams are similar and much steeper than for the pump beam, due to its fundamental absorption. The ESA beam relies mostly on a nonlinear multi photon absorption and is therefore much more sensitive to energy changes (Fig. 3b).

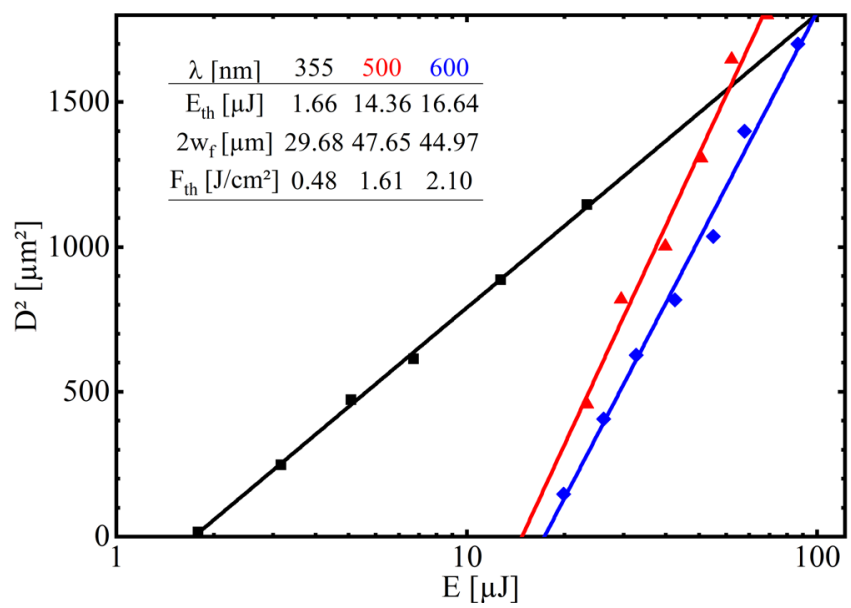

Fig. 2 Semilog plot of $E$ over $D^{2}$ to determine $E_{t h}, 2 w_{f}$, and $F_{t h}$ (attached table) using the method proposed by Liu [33] for the individual pump and ESA beams.

\subsection{ESA based ablation}

Compared to a single photon absorption, for an ESA based ablation process, two laser beams - the pump beam with $\mathrm{E}_{\text {pump }}>\mathrm{E}_{\mathrm{BG}}$ and the ESA beam with $\mathrm{E}_{\mathrm{ESA}}<\mathrm{E}_{\mathrm{BG}}-$ are spatially and temporally super imposed (Fig. 1, 3a). All used $\mathrm{F}_{\text {pump }}$ and $\mathrm{F}_{\mathrm{ESA}}$ were below their respective $\mathrm{F}_{\text {th }}$ and therefore no ablation is seen for the individual pulses (Fig. 2).

The ablation spot diameters $\mathrm{D}$ for different $\Delta \mathrm{t}$ and $\mathrm{F}_{\mathrm{ESA}}$ $(\lambda=500,600 \mathrm{~nm})$ at $\mathrm{F}_{\text {pump }}=0.2 \mathrm{~J} / \mathrm{cm}^{2}$ are shown in Fig. 4. Here, with higher $\mathrm{F}_{\mathrm{ESA}}$ the spot diameters and time window between the pulses, in which an ablation can be observed, increase, due to the increased amount of absorbed energy inside the $\mathrm{ZnO}$ layer. Furthermore, no ablation is seen for negative temporal delays. Without prior excitation of the pump, the ESA beam is hardly absorbed by the $\mathrm{ZnO}$ and cannot contribute to the heating of the material.
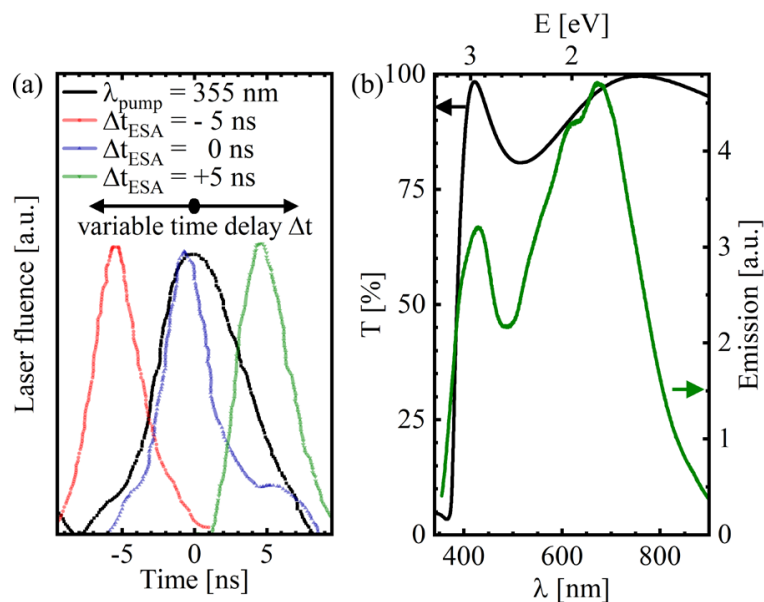

Fig. 3 (a) Temporal delay $\Delta t$ between the pump beam and the

ESA beams for $\Delta \mathrm{t}=-5,0,+5 \mathrm{~ns}$. (b) Transmittance spectrum of the $200 \mathrm{~nm}$ thin $\mathrm{ZnO}$ layer measured with uncoated fused silica as reference material, as well as the emission spectrum with $\lambda=325 \mathrm{~nm}$ as excitation source.

As shown in Fig. 3a, the temporal overlap of the pulses for $\Delta \mathrm{t}=-5$ and $+5 \mathrm{~ns}$ are comparable, but almost no ablation is seen for negative delays and thus, a pure accumulation effect of the energy of both pulses can be excluded, since otherwise the ablation diameters would show a closer resemblance. Additionally, the optimum for a heat and energy accumulation would be expected at $\Delta \mathrm{t}=0 \mathrm{~ns}$ at the respective intensity peak of both pulses. Taking the ESA process into account, the ablation of the material is only possible if the pump beam excites the material first, e.g., by exciting electrons to the conduction band prior to the subsequent ESA beam which can then deposit enough additional energy into the $\mathrm{ZnO}$ layer to exceed the required ablation threshold energy.

Consequently, optimum ESA conditions were observed at $\Delta \mathrm{t} \approx 5 \mathrm{~ns}$, where the highest density of excited carriers is available for the ESA beam. For $\Delta \mathrm{t}<5 \mathrm{~ns}$, only a fraction of the pump beam energy is used. As the temporal pulse overlap further decreases, for $\Delta \mathrm{t}<0 \mathrm{~ns}$ only occasionally small ablation spots were observed due to the insufficient amount of available excited carriers.

On the other hand, the decreasing $\mathrm{D}$ for $\Delta \mathrm{t}>5 \mathrm{~ns}$ is caused by the relaxation of excited carriers. Here, besides the conduction band electrons, deep (defect) levels serve as additional potential absorption centers for an ESA, generally characterized by longer lifetimes, extending the processing window [38-40] and are a key driving force for ESA based ablation. For $\lambda_{500}$ a processing window with pulse delays above $300 \mathrm{~ns}$ is seen (Fig. 4a), compared to less than $70 \mathrm{~ns}$ for $\lambda_{600}$ (Fig. 4b). In the latter case, the higher wavelengths and therefore lower photon energies restrict the ESA primarily to intraband transitions with a short fluorescence lifetime of $<10 \mathrm{~ns}[38,39]$ or a limited fraction of energy (defect) levels close to the conduction band, as the ESA beam energy needs to be sufficient to reach the conduction band from these energy levels, as otherwise no ESA is allowed and the ESA beam is mostly transmitted. Due to the higher photon energy of $\lambda_{500}$, the amount of available absorption centers 

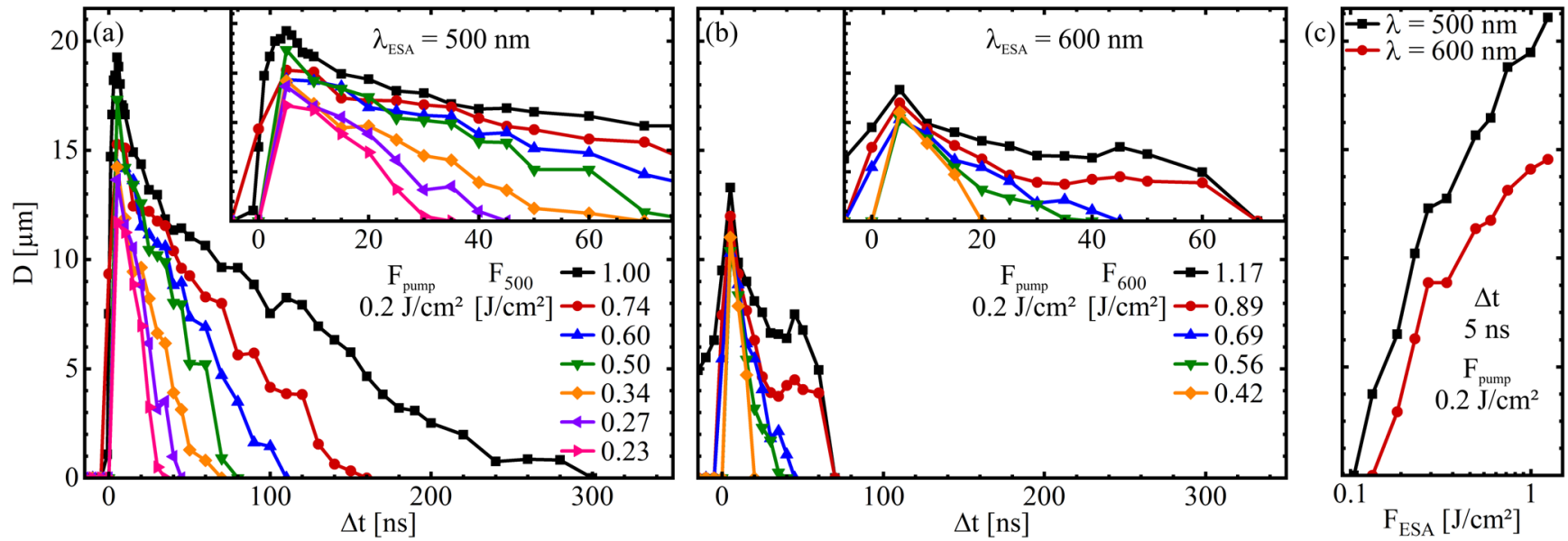

Fig. 4 (a, b) Ablation diameters at different pulse delays and $\mathrm{F}_{\mathrm{ESA}}$ for (a) $\lambda_{\mathrm{ESA}}=500 \mathrm{~nm}$ and (b) $\lambda_{\mathrm{ESA}}=600 \mathrm{~nm}$ at constant $\mathrm{F}_{\text {pump }}=0.2 \mathrm{~J} / \mathrm{cm}^{2}$. (c) $D$ for different $\mathrm{F}_{\mathrm{ESA}}$ at constant $\Delta \mathrm{t}=5 \mathrm{~ns}$ and $\mathrm{F}_{\text {pump }}=0.2 \mathrm{~J} / \mathrm{cm}^{2}$.

able to absorb photons into the conduction band by a one photon ESA are increased. The highest amount of deep level defects is located in the broad range of $\lambda \sim 500-800 \mathrm{~nm}$ where the strongest emission is seen (Fig. 3b), confirming the extended fluorescent lifetime of these defect levels $[39,40]$.

It is obvious, that the ablation close to the optimum delay differs far less than for higher delays. Here, the pulses still overlap, creating an excess of conduction band electrons which in turn reduces the importance of defect level, only usable to higher energetic photons. Thus, a decreased $\lambda$-dependence is observed. The ablation spots for different $\mathrm{F}_{\mathrm{ESA}}$ at the optimum $\Delta \mathrm{t}=5 \mathrm{~ns}$ is shown in Fig. $4 \mathrm{c}$.

The ESA based process is characterized by an almost equal efficiency when compared to single beam ablation (Fig. 2, 4). As shown in Fig. $5 \mathrm{~d}$, with $\mathrm{F}_{\text {pump }}=0.21 \mathrm{~J} / \mathrm{cm}^{2}$ and $\mathrm{F}_{\mathrm{ESA}}=0.19 \mathrm{~J} / \mathrm{cm}^{2}$, the combination of both laser energies $\left(\mathrm{F}=0.4 \mathrm{~J} / \mathrm{cm}^{2}\right)$ is still slightly below the required threshold for an ablation using only a single pump pulse (Fig. 2).

Besides this efficiency improvement, the ablation quality is significantly enhanced as well and the spot diameters are decreased. The ablation area is restricted by the superposition of both pulses, limiting the ablation to the mostly uniform high intensity center of the pulses by threshold effects [23]. Fig. 5 shows selected ablation spots for the different wavelengths witch comparable spot diameters. The pump beam (Fig. 5a) hits the $\mathrm{ZnO}$ layer first (Fig. 1) and due to its high fundamental absorption, the layer is uniformly ablated without damaging the fused silica.

In comparison, the ESA beams cause an irregular ablation. Furthermore, the fused silica was damaged as seemingly most of the energy was absorbed at the interface between fused silica and $\mathrm{ZnO}$ (Fig. 5 b,c). An increased concentration of irregularities and defects are expected in these regions, increasing the absorption and leading to an abrupt heating of this area [40]. Compared to the pump beam, the ESA beams pass the fused silica and therefore this region first with most of the laser energy absorbed here causing these irregular ablations. By decreasing $\mathrm{F}_{\mathrm{ESA}}$ this process is suppressed to a certain degree, as the energy to damage the fused silica substrate is insufficient, but the resulting spots are not circular partially disconnected. Here, areas with an inhomogeneous distribution of defects and absorption centers seem to allow only a partial, spatially confined ablation.
The ESA based ablation allows for highly uniform and circular ablation spots (Fig. 5d) with a clean separation of the $\mathrm{ZnO}$ layer and the fused silica substrate devoid of any substrate damage at comparable energy conditions.
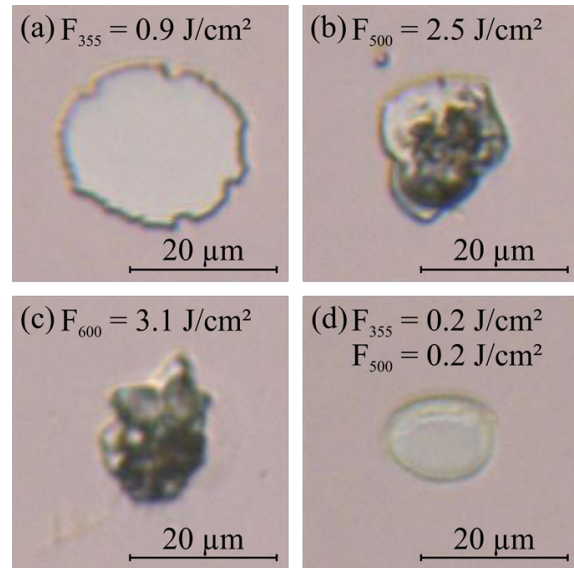

Fig. 5 Microscopic images of ablation spots for a separate single pulse with (a) $F_{\text {pump }}=0.9 \mathrm{~J} / \mathrm{cm}^{2}$, (b) $F_{500}=2.5 \mathrm{~J} / \mathrm{cm}^{2}$, (c) $\mathrm{F}_{600}=3.1 \mathrm{~J} / \mathrm{cm}^{2}$. (d) Improved ablation quality by an ESA based ablation process with $F_{\text {pump }}=0.2 \mathrm{~J} / \mathrm{cm}^{2}$, $\mathrm{F}_{500}=0.2 \mathrm{~J} / \mathrm{cm}^{2}$, and $\Delta \mathrm{t}=5 \mathrm{~ns}$.

\section{Conclusion}

In the present study we built on the previous findings for the hybrid laser processing of an ESA based ablation with $\lambda_{\text {pump }}=355 \mathrm{~nm}$ and $\lambda_{\mathrm{ESA}}=600 \mathrm{~nm}$ and extended the studied parameters to $\lambda_{\mathrm{ESA}}=500$, expanded the $\Delta \mathrm{t}$-ablation range above $300 \mathrm{~ns}$, and expand the $\mathrm{F}_{\mathrm{ESA}}$-range. The quality of the ablation spots is improved and at comparable laser energy conditions. Furthermore, the finding helped to better understand the ESA process by linking the results to the $\lambda$-dependent absorption of deep energy levels, improving the usable pulse delay- and $\mathrm{F}_{\mathrm{ESA}}$-range. Based on these findings, future investigations on a combination of STED and ESA with regards to SDW-processes are made possible.

\section{Acknowledgments}

The authors gratefully acknowledge funding of the project by the DFG. We also thank the groups of Prof. Ronning and Prof. Dietzek for experimental support. 


\section{References}

[1] S. W. Hell, K. I. Willig, M. Dyba, S. Jakobs, L. Kastrup, and V. Westphal: "Handbook of biological confocal microscopy", ed. by J. B. Pawley, (SpringerScience + Business Media, New York, 2006), p.571.

[2] C. Mack: "Fundamental Principles of Optical Lithography: The Science of Microfabrication", ed. by C. Mack, (John Wiley \& Sons, Chichester, 2008), p.1.

[3] D. P. Sanders: Chem. Rev., 110, (2010) 321.

[4] A. C. Assafrao, N. Kumar, A. J. H. Wachters, S. F. Pereira, and H. P. Urbach: Jpn. J. Appl. Phys., 53, (2014) 042001.

[5] M. Wegener: Micro Electro Mechanical Systems (MEMS), 2011 IEEE 24th International Conference on Micro Electro Mechanical Systems, Vol. (2011) 1.

[6] A. Frolich, J. Fischer, T. Zebrowski, K. Busch, and M. Wegener: Adv. Mater., 25, (2013) 3588.

[7] J. Fischer and M. Wegener: Laser Photon. Rev., 7, (2013) 22.

[8] J. E. E. Baglin: Appl. Surf. Sci., 258, (2012) 4103.

[9] E. Ozbay: Science, 311, (2006) 189.

[10] S. Wong, M. Deubel, F. Pérez-Willard, S. John, G. A. Ozin, M. Wegener, and G. von Freymann: Adv. Mater., 18, (2006) 265.

[11] V. R. Manfrinato, L. Zhang, D. Su, H. Duan, R. G. Hobbs, E. A. Stach, and K. K. Berggren: Nano Lett., 13, (2013) 1555.

[12] I. Utke: Nanofabrication using focused ion and electron beams : principles and applications, Oxford University Press, New York, 2012.

[13] T. C. Chong, M. H. Hong, and L. P. Shi: Laser Photon. Rev., 4, (2010) 123.

[14] A. Mallik, W. Vansumere, J. Ryckaert, A. Mercha, N. Horiguchi, S. Demuynck, J. Bömmels, T. Zsolt, G. Vandenberghe, K. Ronse, A. Thean, D. Verkest, H. Lebon, and A. Steegen: Vol. 8679, (2013) 86792Y.

[15] A. F. G. Leontowich and A. P. Hitchcock: Appl. Phys. A, 103, (2011) 1.

[16] J. de Boor, N. Geyer, J. V. Wittemann, U. Gosele, and V. Schmidt: Nanotechnology, 21, (2010) 095302.

[17] J. de Boor, N. Geyer, U. Gösele, and V. Schmidt: Opt. Lett., 34, (2009) 1783.

[18] G.-Y. Jung, E. Johnston-Halperin, W. Wu, Z. Yu, S.-Y. Wang, W. M. Tong, Z. Li, J. E. Green, B. A. Sheriff, A. Boukai, Y. Bunimovich, J. R. Heath, and R. S. Williams: Nano Lett., 6, (2006) 351.

[19] T. F. Scott, C. J. Kloxin, D. L. Forman, R. R. McLeod, and C. N. Bowman: Journal of Materials Chemistry, 21, (2011) 14150.

[20] M. Malinauskas, M. Farsari, A. Piskarskas, and S. Juodkazis: Phys. Rep., 533, (2013) 1.

[21] L. Li and J. T. Fourkas: Materials Today, 10, (2007) 30.

[22] K.-i. Kawamura, M. Hirano, T. Kurobori, D. Takamizu, T. Kamiya, and H. Hosono: Appl. Phys. Lett., 84, (2004) 311.

[23] F. Korte, J. Serbin, J. Koch, A. Egbert, C. Fallnich, A. Ostendorf, and B. Chichkov: Appl. Phys. A, 77, (2003) 229.

[24] R. R. Gattass and E. Mazur: Nat Photon, 2, (2008) 219.

[25] G. Cox and C. J. Sheppard: Microsc Res Tech, 63, (2004) 18.
[26] R. Heintzmann and G. Ficz: Brief Funct Genomic Proteomic, 5, (2006) 289.

[27] L. Fritzky and D. Lagunoff: Anal Cell Pathol (Amst), 36, (2013) 5.

[28] T. A. Klar, R. Wollhofen, and J. Jacak: Physica Scripta, T162, (2014) 014049.

[29] R. Wollhofen, J. Katzmann, C. Hrelescu, J. Jacak, and T. A. Klar: Opt. Express, 21, (2013) 10831.

[30] J. Fischer and M. Wegener: Opt. Mater. Express, 1, (2011) 614.

[31] W. Zhou, D. Bridges, R. Li, S. Bai, Y. Ma, T. Hou, and A. Hu: Sci. Lett. J, 5, (2016) 228.

[32] S. Engel, C. Wenisch, S. Gräf, and F. Müller: Proc. SPIE, Vol. 11268, (2020) 112680D.

[33] J. Liu: Opt. Lett., 7, (1982) 196.

[34] J. Tauc, R. Grigorovici, and A. Vancu: Physica Status Solidi B, 15, (1966) 627.

[35] B. E. Sernelius, K.-F. Berggren, Z.-C. Jin, I. Hamberg, and C. G. Granqvist: Phys. Rev. B, 37, (1988) 10244.

[36] D. Mardare, M. Tasca, M. Delibas, and G. Rusu: Appl. Surf. Sci., 156, (2000) 200.

[37] T. Wang, S. Zheng, W. Hao, and C. Wang: Surf. Coat. Technol., 155, (2002) 141.

[38] A.-H. Tang, Z.-X. Mei, Y.-N. Hou, and X.-L. Du: Chinese Physics B, 27, (2018) 117802.

[39] N. S. Han, H. S. Shim, J. H. Seo, S. Y. Kim, S. M. Park, and J. K. Song: J. Appl. Phys., 107, (2010) 084306.

[40] A. Layek, B. Manna, and A. Chowdhury: Chem. Phys. Lett., 539, (2012) 133.

(Received: June 25, 2020, Accepted: October 5, 2020) 